\title{
Guided Image Filter and Adaptive Histogram Specification based DBLA for Enhancing Remotely Sensed Images
}

\author{
Richa Bhatia \\ M.Tech Research Scholar
}

\author{
Jagdeep Singh Aulakh \\ Assistant Professor
}

\begin{abstract}
The fundamental reason of contrast enhancement is to bring out detail that is hidden in an image that is covered up in a picture or to expand contrast in a low contrast picture. This paper presents a literature survey on some of the image Enhancement techniques for improving images like histogram equalization, global histogram equalization, bi-histogram equalization, dualistic sub-image histogram equalization, Spatial Domain Method and Frequency Domain Method etc. The overall objective of this paper is to evaluate the short comings in earlier techniques of image enhancement.
\end{abstract}

\section{Keywords}

Contrast Enhancement, HE

\section{INTRODUCTION}

Image Processing is some kind of signal processing for which the input value is a video, voice or images; and then relative outcome of image processing may be a video, voice, an images or a set of parameters that are re1ated to the input image. Generally this technique can be used as a twodimensional signal. Image processing can be separated into two categories:

\subsection{Analog Image Processing}

It is complete on analog signals. Analog Image processing can be proceed on $2 \mathrm{D} \mathrm{M} * \mathrm{~N}$ dimensional analog signals. In this category of processing method, image is handled by varying the electrical signal. The most usual picture is the television image.

\subsection{Digital Image Processing}

This processing technique allocates with building a digital system that proceeded on digital images.

\section{IMAGE ENHANCEMENT}

Image enhancement is really a preprocessing portion of a lot of graphic running programs. Principle goal of graphic enhancement would be to enhance the interpretability or maybe perception of facts inside images regarding people, so they can source much better suggestions regarding some other computerized graphic running approaches. There are many causes of inferior of a video including as an illustration distortion staying unveiled because of the imaging systems, too little expertise of the agent or maybe the actual damaging outside conditions in the time graphic buy. Moreover, Image enhancement contains strength along with contrast tricks, noise decline, edge sharpening along with blocking, and so forth. Form a contrast Improvement is actually committed to the specific situation of improving the actual contrast in a very video to generate numerous features more rapidly understood. Form a contrast of a video depends on it is dynamic array, which is outlined for the reason that big difference among most competitive along with best strength degree. Form a contrast enhancement approaches possess numerous software regions regarding enhancing the actual graphic quality of lower contrast images. Form a contrast enhancement is actually one of several important study difficulties of graphic enhancement.

\section{CONTRAST ENHANCEMENT TECHNIQUES}

Following are few contrast enhancement techniques has been described below.

\subsection{Histogram equalization}

This technique usually escalates the global contrast of several images, especially once the utilizable data of the image is represented by nearby contrast values. All the way through this adjustment, the intensities could be better distributed on the histogram. This happens for parts of lower local contrast to improve a larger contrast. Histogram equalization accomplishes this by successfully spreading out the absolutely frequent intensity values. The strategy is advantageous in images with backgrounds and foregrounds which are generally bright or both dark. Specifically, the strategy can lead to raised views of bone structure in x-ray images, and raised detail in photographs which are over or under-exposed. A vital advantageous asset of the strategy is that it's a reasonably straightforward technique and an invertible operator. So the idea is that, if the histogram equalization function is famous, then an original histogram could be recovered. The calculation is not computationally intensive. A disadvantage of the strategy is that it's indiscriminate. It might boost the contrast of background noise, while decreasing the usable signal. In scientific imaging where spatial correlation is more important than intensity of signal, the tiny signal to noise ratio usually hampers visual detection. Histogram equalization often produces unrealistic effects in photographs; however it is extremely helpful for scientific images like thermal, satellite or x-ray images, exactly the same class of images that user would apply false-color to. Also histogram equalization can produce undesirable effects when positioned on images with low color depth. As an illustration, if positioned on 8-bit image displayed with 8-bit gray-scale palette it'll further reduce color depth of the image. Histogram equalization will continue to work the most truly effective when positioned on images with higher color depth than palette size, like continuous data or 16-bit grayscale images.

\subsection{Global Histogram Equalization}

Let us suppose that $\mathrm{X}=\{\mathrm{X}(\mathrm{i}, \mathrm{j})\}$ denotes a digital image, where $X(i, j)$ denotes the gray level of the pixel at $(i, j)$ place. The total number of the image pixels is $\mathrm{n}$, and the image intensity is digitized into $\mathrm{L}$ levels that are $\{X 0, X 1$, $\left.X 2 \ldots . X_{L-1}\right\}$. So it is obvious that $\forall$ $\mathrm{X}(\mathrm{i}, \mathrm{j}) \in\left\{X 0, X 1, X 2, \ldots . X_{L-1}\right\}$.Suppose $n k$ denotes the total number of pixels with grey level of $X_{k}$ in the image, then the probability density of $X_{k}$ will be 


$$
P\left(X_{k}\right)=\frac{n_{k}}{n}, k=0,1, \ldots \ldots L-1
$$

The relationship between $\mathrm{p}\left(X_{k}\right)$ and $X_{k}$ is defined as the probability density function (PDF), and the graphical appearance of PDF is known as the histogram. Based on the image's PDF, its cumulative distribution function is defined as

$C\left(r X_{k}\right)=\sum_{j=0}^{L-1} p\left(X_{j}\right)=\sum_{j=0}^{L-1} \frac{n_{k}}{n}$

Where $\mathrm{k}=0,1, \ldots \ldots \ldots \ldots, \mathrm{L}-1$, and it is obvious that $\mathrm{c}\left(X_{L-1}\right)=1$. The output mean of the HE does not take the mean brightness of the original image into account.

\subsection{Bi-Histogram Equalization}

These strategies separate input histogram into two subsections. These two elements equalized severally. During this methodology the factors accustomed selected the threshold for separation denoted by. $\epsilon$............Based on the threshold the input image $X$ are often rotten into sub-images into two sub-images[8].This methodology divides the image histogram into two separate parts as shown in Fig.1. In this method, the separation intensity is conferred by the input mean brightness value, which can be the average intensity of most pixels that construct the input image. When this separation method, these two histograms are severally equal. Using this method, the mean brightness of the resultant image can lie between the input mean and also the middle grey level.

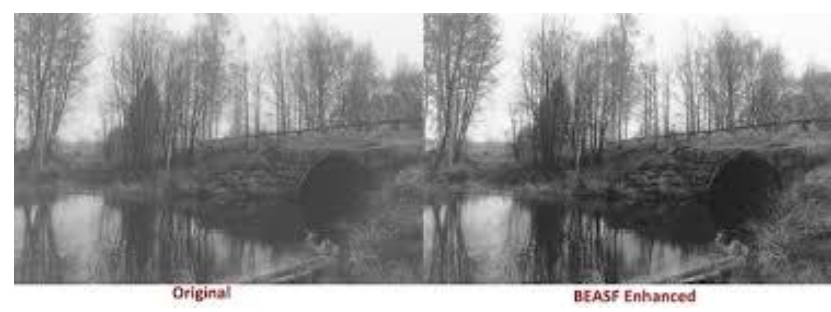

Figure 1: Input and Output image

\subsection{Dualistic Sub-Image Histogram Equalization}

Subsequently the basic concepts employed by the BBHE method of decomposing the new image into sub-images and hence equalize the histograms of the sub-images individually, proposed the thus known as equal area dualistic sub-image HE technique. Rather than decomposing the image supported on its mean grey level, the DSIHE technique decompose the images that aims at the maximization of the Shannon's entropy of the output image of decomposing the first image into two sub-images and hence equalize the histograms of the sub-images individually.

\subsection{Spatial Domain Method}

Spatial domain method directly work on pixel. The procedure can be composed as $g(i, j)=T[f(i, j)]$, where $g$ is the outcome, $f$ is the input and $\mathbf{T}$ is a process on $f$ defined above a few neighborhood of $(i, j)$.This technique is using the following equation.

$$
g(i, j)=f(i, j) * h(i, j)
$$

\subsection{Frequency domain method}

Frequency domain method process on the Fourier Transform. Frequency domain picture improvement is clear-cut. The frequency filters established an image in the frequency domain. This category filtering method is very straightforward.

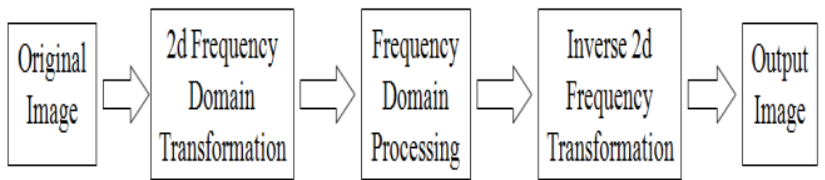

Figure 2: Frequency Domain Method Process

- Transform the picture into the Fourier domain.

- Multiply the image or picture by the filter.

- Take the inverse transform of the picture.

\section{TECHNIQUES USED BY SPATIAL AND FREQUENCY DOMAIN METHODS}

Following are three techniques of spatial and frequency domain methods.

\subsection{Point operations}

Where every pixel is customized according to a mainly equation that is not based on further pixel values.

\subsection{Mask operations}

Where each pixel is customized according to the values of pixel's neighbors (Using convolution theory).

\subsection{Global operations}

When all the pixels value in a picture are taken into concern.

\section{LITERTURE SURVEY}

Aimi Salihah et al. [1] offered a couple of period system to be able to acquire an entirely segmented unusual whitened blood cell phone and also nucleus inside severe leukaemia photographs. In the first period, the particular several distinction enhancement methods which might be partially distinction, shiny stretches and also black stretches had been accustomed to improve the picture top quality. Form a contrast enhancement methods enhanced the spot connected with attention connected with severe leukaemia for easing the particular segmentation course of action. Over the following period, picture segmentation dependent on HSI coloration space have been recommended. Anbarjafari et al. [2] provides light troubles happen to be a significant problem in lots of picture digesting software. The pattern in the histogram by using an picture features meaningful functions; for this reason inside the technique of light enhancement, it is crucial not to destroy such data. A strategy to increase picture light making use of Gaussian distribution mapping have been recommended which usually in addition retains the details laid about the pattern in the histogram about the first picture. Sundaram, L. [3] offered a Modified Form a contrast Constrained Adaptive Histogram Equalization. Histogram equalization is actually an efficient and also basic way of distinction enhancement. The original histogram equalization normally ends up with excessive distinction enhancement due to inadequate command upon the amount of enhancement. The Histogram Modified Form a contrast Constrained Adaptive Histogram Equalization tunes the total amount connected with distinction enhancement, which regularly provides the particular resulting picture a powerful distinction and also delivers the region facts for much more applicable design. K. Akila et al. [4] provides discussed which distinction enhancement is usually an crucial difficulty in the field of mammographic picture digesting. Oblique distinction enhancement entails inside enhancing histogram in the picture. Histogram equalization (HE) is the most straightforward indirect distinction enhancement process and 
that is popular for distinction enhancement. A lot of options connected with HE tend to be recommended until now. Contrast of such methods is actually substantially essential inside choosing the right algorithm for enhancement and additional digesting. Ke, Wei-Min et al. [5] recommended a progressive picture enhancement platform which includes bilateral tone realignment and also saliency-weighted distinction enhancement techniques. In contrast to almost all curve-based worldwide distinction enhancement techniques. Lui and also Xiwen [6] get discussed which due to shortcomings in the traditional unclear enhancement algorithms, numerous enhancements happen to be recommended. Inside the improved upon algorithm, the particular member's program characteristics and also unclear enhancement user are made connected with piecewise continual characteristics, and also the picture is actually split straight into a couple of parts by simply OTSU technique, the first is excessive greyish area, one other is actually lower greyish area, pixels in the excessive greyish area tend to be enhanced, and also pixels in the lower greyish area tend to be decreased. Jha et al. [7] recommended a nonlinear nondynamic stochastic resonance-based way of enhancement connected with black and also lower distinction photographs. A decreased distinction picture is actually treated as being a sub-threshold sign and also noise-enhanced sign digesting lies upon improve its distinction. The recommended process exclusively employs improvement connected with additional sounds to help reduce the effects of the particular affectation connected with central sounds of the lower distinction picture. M.D Jadhav and R. L. Patil [8] provides discussed which purchase connected with excessive satellite images gets hard a result of the guitar restriction and also imperfect image resolution optics. Small spatial quality satellite for personal computer photographs comprise many merged pixels which usually decay the particular diagnosis and also identification performance inside civil and also armed forces software. Satellite picture quality enhancement making use of DyadicInteger coefficients based bi-orthogonal wavelet filtration systems have been recommended. Dyadic-integer-coefficient based wavelet filtration systems tend to be produced by the particular design of the half-band polynomial. Xu, Hongteng et al. [9] recommended a histogram-based product for distinction enhancement;. In line with the examination with regards to the particular interactions connected with histogram with distinction, these people start a design which usually realized distinction enhancement by simply an perfect transform connected with histogram, presented a couple of metrics termed distinction obtain and also non linearity connected with transform to help gauge the potency of enhancement and also the seriousness connected with distortion attributable to enhancement respectively. Verbesselt et al. [10] provides discussed which close to real-time keeping track of connected with environment agitations is crucial for speedily examining and also handling has effects on upon carbon dioxide dynamics, biodiversity, and also socioecological techniques. Satellite distant sensing makes it possible for cost-effective and also appropriate keeping track of in recurrent occasion measures in excess of substantial parts. Satellite picture occasion combination of plant life greenness provides a worldwide record connected with terrestrial plant life productiveness within the last decades. Chouhan et al. [11] recommended a dynamic stochastic resonance based process inside spatial domain for that enhancement connected with dark- and also low-contrast photographs. Stochastic resonance happening in that the particular performance connected with one thing may be improved upon by simply improvement connected with sounds. DSR is actually put on within a iterative style by simply correlating the particular bi stable method guidelines of the double-well potential while using the depth ideals of the low-contrast picture. The best possible production is actually guaranteed by simply adaptive computation connected with performance metrics - comparable distinction enhancement component, perceptual top quality steps and also coloration enhancement component. As compared to the prevailing enhancement methods. Petitjean et al. [12] provides discussed which satellite picture occasion collection are becoming progressively more readily available all of which will keep on to do so in the returning many years because of the particular introduction connected with space missions which usually purpose in delivering protection in the Soil every single 7 days with excessive spatial quality. When it comes to optical images, you'll be able to produce area employ and also protect alter maps with precise nomenclatures. Nonetheless, due to meteorological phenomena. A technique for picture occasion collection examination have been offered which usually has the ability to manage irregularly sampled collection. C. Reshmalakshmi and M. Sasikumar [13] managed the latest distinction enhancement algorithm, which usually maps components coming from pixel aircraft to help member's program aircraft also to enhancement/transform aircraft. Mistakes connected with present distinction enhancement methods tend to be rectified with the assistance of a statistical application termed `Fuzzy fixed i. These unclear models may be carved to regulate the particular uncertainty and/or vagueness associated with photographs. To be able to gauge the particular performance, this brand new algorithm is actually put on upon unique photographs and also handful of assessment guidelines had been worked out. Melody et al. [14] provides discussed which to be able to improve the local feature's describing capacity and also enhance the group performance connected with high-resolution (HR) satellite photographs, satellite picture landscape have been offered to help group technique which take advantage of spatial data connected with local element. 1st, the particular spatial pyramid coordinating product (SPMM) is actually implemented to help encode spatial data connected with local element. Subsequently, photographs tend to be showed from the local element descriptors and also encoding data. Ultimately, the particular service vector unit (SVM) classifier must be used to help classify picture clips. G. Maragatham et al. [15] recommended an algorithm to help product photographs displaying its local distinction gauge, to help classify and also recognize associated with the particular photographs possessing unique distinction degree. The insight picture is actually grouped either because lower distinction or excessive distinction picture by way of the particular product. If your grouped picture is actually lower distinction will probably be enhanced by way of the particular Stochastic Resonance principle. The outcomes exposed that the recommended automated course of action boosts the reduced distinction picture far better when compared with standard enhancement techniques. McRoberts et al. [16] provides discussed which methodical technique have been indicated because possessing a couple of specific elements, Development and also Validation. In contrast to Development, Validation has a official reasoning in whose principles need to be carefully put into practice to produce appropriate methodical inferences. Particularly, when inferences use trial info, the foundations in the reasoning connected with Validation call for tests connected with opinion and also perfection. Therefore, satellite image-based maps which shortage such tests for guidelines connected with populations shown from the maps might be connected with tiny energy for methodical inference; primarily, they usually are just pretty pictures. Cao Gang et al. [17] recommended a 
couple of new algorithms to help diagnose the particular distinction enhancement engaged manipulations inside digital photographs. 1st, these people predicated about the diagnosis connected with worldwide distinction enhancement positioned on the particular earlier JPEG-compressed photographs, which might be common inside real software. Minute, these people recommended to distinguish the particular amalgamated picture made by enforcing distinction realignment upon each one or each resource parts. Iqbal et al. [18] Quality enhancement (RE) techniques are afflicted by the particular negative aspect connected with dropping highfrequency contents. The discrete-wavelet-transform-based (DWT) RE scheme creates artifacts. Some sort of waveletdomain approach dependent on dual-tree difficult wavelet transform (DT-CWT) and also non local indicates (NLM) is actually recommended for RE in the satellite photographs

\section{PROPOSED ALGORITHM}

In this algorithm by applying different techniques of image enhancement on remote sensing images, the result will be shown in the form of enhanced image.

Step1. Select an input image.

Step2. Apply discrete wavelet transform on an input image.

Step3. Then dominant brightness level analysis.

Step4. Divide an image based on dominant brightness level analysis into three intensity (low, middle, high) layers.

Step5. Then apply adaptive intensity transfer function on each layer and do boundary smoothing after smoothing weighting map estimation method applied on it.

Step6. After that Adaptive histogram equalization technique has been applied.

Step7. Then image can be fused means to combine the decomposed image into a single one.

Step8. Inverse discrete wavelet transform method has been applied on it

Step9. Now illuminant enhancement will come in action to balance the colors and thus will reduce the color affects due to the discrete wavelet transform (DWT).

Step10. Then apply the method of guided image filter to reduce the noise in an image.

Step11. Result has been shown in the form of contrast enhanced image.

\section{RESULTS AND DISCUSSION}

The experiment has taken on different satellite images. Satellite image is input in simulation environment i.e. Matlab 2013 and results on different images has been shown in figure 3 and 4 respectively and values of different parameters also be calculated parameters like Mean error ratio (MER), Peak signal noise ratio (PSNR) and Root mean square error (RMSR).

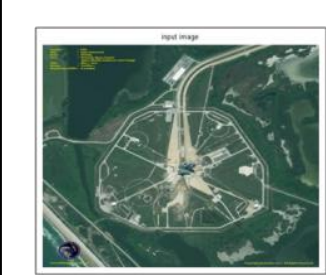

(a) Input Image

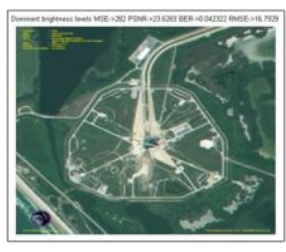

(b) Output Image

(Dominant Brightness)

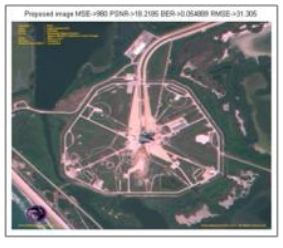

(c) Output Image (Proposed Image)

Figure 3: Input and Output Images

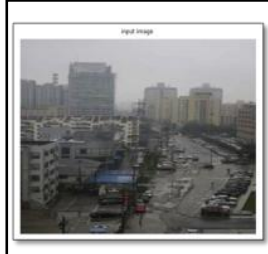

(a) Input Image

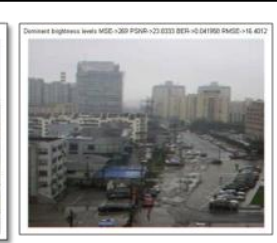

(b) Output Image

(Dominant Brightness)

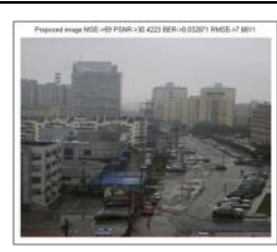

(c) Output Image (Proposed Image)

Figure 4: Input and Output Images

Table 1. Mean Square Error

\begin{tabular}{|c|c|c|}
\hline Images & Proposed MSE & Existing MSE \\
\hline 1 & 59 & 269 \\
\hline 2 & 16 & 246 \\
\hline 3 & 150 & 314 \\
\hline 4 & 169 & 481 \\
\hline 5 & 100 & 319 \\
\hline 6 & 145 & 661 \\
\hline 7 & 86 & 354 \\
\hline 8 & 60 & 254 \\
\hline 9 & 572 & 1428 \\
\hline 10 & 105 & 362 \\
\hline 11 & 232 & 889 \\
\hline 12 & 154 & 487 \\
\hline 13 & 283 & 979 \\
\hline 14 & 147 & 451 \\
\hline 15 & 351 & 699 \\
\hline
\end{tabular}

Table 1 contains the values of Mean Square Error (MSE) of the proposed and existing algorithm corresponding to the 15 different images. The MSE of the proposed algorithm has less value than existing algorithm. According to these values graph 1 has plotted. 


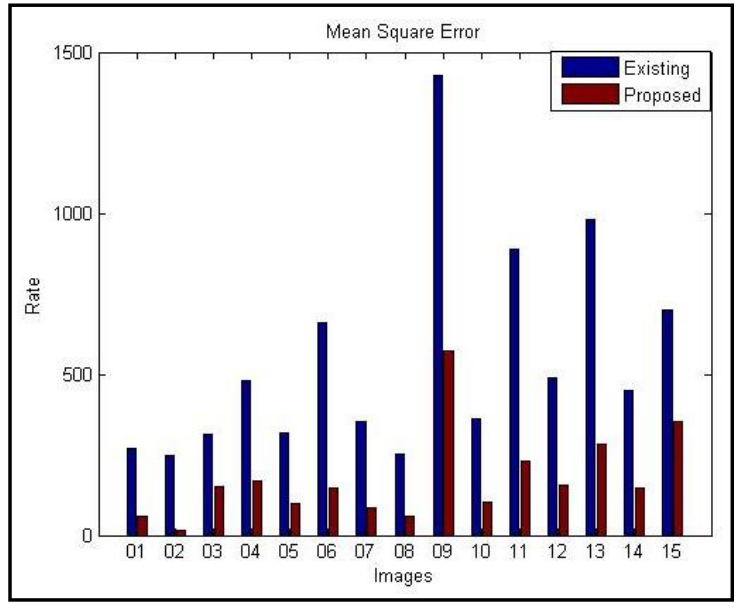

Graph 1: Mean Square Error

Table 2 contains the values of Peak Signal Noise Ratio (PSNR) of the proposed and existing algorithm corresponding to the 15 different images. The PSNR of the proposed algorithm has more value than existing algorithm. According to these values graph 2 has plotted.

Table 2. Peak Signal Noise Ratio

\begin{tabular}{|l|l|l|}
\hline Images & Proposed PSNR & Existing PSNR \\
\hline 1 & 30.4223 & 23.8333 \\
\hline 2 & 36.0896 & 24.2215 \\
\hline 3 & 26.3699 & 23.1615 \\
\hline 4 & 25.8519 & 21.3094 \\
\hline 5 & 28.1308 & 23.0929 \\
\hline 6 & 26.5171 & 19.9288 \\
\hline 7 & 28.7858 & 22.6041 \\
\hline 8 & 30.3493 & 24.0825 \\
\hline 9 & 20.5568 & 15.5110 \\
\hline 10 & 27.9189 & 22.5437 \\
\hline 11 & 24.4759 & 18.6418 \\
\hline 12 & 26.2556 & 21.2555 \\
\hline 13 & 23.6129 & 18.2230 \\
\hline 14 & 26.4576 & 21.5890 \\
\hline 15 & 22.6777 & 19.6860 \\
\hline
\end{tabular}

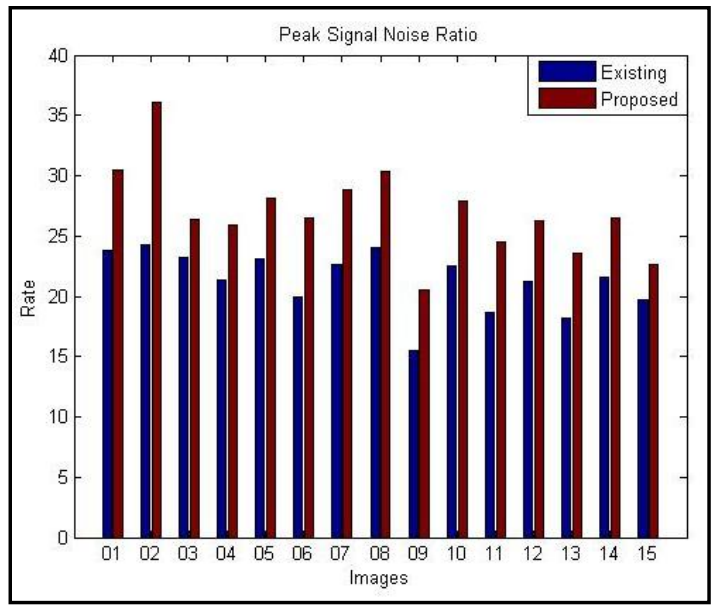

Graph 2: Peak Signal Noise Ratio
Table 3. Root Mean Square Error

\begin{tabular}{|l|l|l|}
\hline Images & Proposed RMSE & Existing RMSE \\
\hline 1 & 7.6811 & 16.4012 \\
\hline 2 & 4.0010 & 15.6844 \\
\hline 3 & 12.2474 & 17.7200 \\
\hline 4 & 13.0001 & 21.9317 \\
\hline 5 & 10.0011 & 17.8606 \\
\hline 6 & 12.0416 & 25.7099 \\
\hline 7 & 9.2736 & 18.8944 \\
\hline 8 & 7.7460 & 15.9374 \\
\hline 9 & 23.9165 & 42.7551 \\
\hline 10 & 10.2470 & 19.0263 \\
\hline 11 & 15.2315 & 29.8161 \\
\hline 12 & 12.4097 & 22.0681 \\
\hline 13 & 16.8226 & 31.2890 \\
\hline 14 & 12.1244 & 21.2368 \\
\hline 15 & 18.7350 & 26.4389 \\
\hline
\end{tabular}

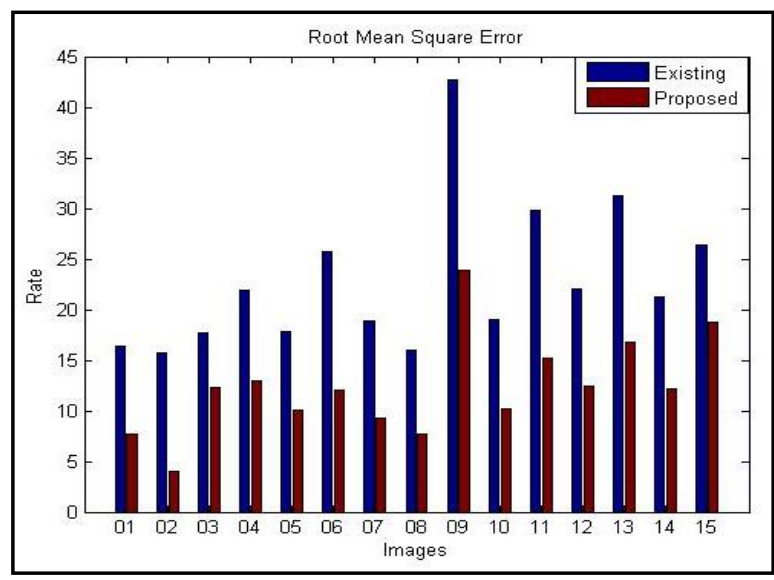

Graph 3: Root Mean Square Error

Table 3 contains the values of Root Mean Square Error (RMSE) of the proposed and existing algorithm corresponding to the 15 different images. The RMSE of the proposed algorithm has less value than existing algorithm. According to these values graph 3 has plotted.

\section{CONCLUSION AND FUTURE SCOPE}

In this paper, a survey on some of the image Enhancement techniques for improving images has been done. Moreover a comparison table has been developed which shows the various techniques like Mean error ratio (MER), Peak signal noise ratio (PSNR) and Root mean square error (RMSR), their features and limitations. Experimental results have been concluded that proposed technique performs better than existing. Therefore, in near future, new integrated Contrast Enhancement technique for satellite Images using Dominant Brightness Level Analysis can be developed for better results.

\section{REFERENCES}

[1] Aimi Salihah, A. N., M. Y. Mashor, Nor Hazlyna Harun, Azian Azamimi Abdullah, and H. Rosline. "Improving colour image segmentation on acute myelogenous leukaemia images using contrast enhancement techniques." In Biomedical Engineering and Sciences (IECBES), 2010 IEEE EMBS Conference on, pp. 246251. IEEE, 2010.

[2] Anbarjafari, Gholamreza, Adam Jafari, Mohammad Naser Sabet Jahromi, Cagri Ozcinar, and Hasan Demirel. "Image illumination enhancement with an objective no- 
reference measure of illumination assessment based on Gaussian distribution mapping." Engineering Science and Technology, an International Journal (2015).

[3] Sundaram, M., K. Ramar, N. Arumugam, and G. Prabin. "Histogram based contrast enhancement for mammogram images." In Signal Processing, Communication, Computing and Networking Technologies (ICSCCN), 2011 International Conference on, pp. 842-846. IEEE, 2011.

[4] Akila, K., L. S. Jayashree, and A. Vasuki. "Mammographic Image Enhancement Using Indirect Contrast Enhancement Techniques-A Comparative Study." Procedia Computer Science 47 (2015): 255-261.

[5] Ke, Wei-Ming, Chih-Rung Chen, and Ching-Te Chiu. "BiTA/SWCE: Image enhancement with bilateral tone adjustment and saliency weighted contrast enhancement." Circuits and Systems for Video Technology, IEEE Transactions on 21, no. 3 (2011): 360-364.

[6] Liu, Xiwen. "An Improved Image Enhancement Algorithm Based on Fuzzy Set." Physics Procedia 33 (2012): 790-797.

[7] Jha, Rajib Kumar, Rajlaxmi Chouhan, and P. K. Biswas. "Noise-induced contrast enhancement of dark images using non-dynamic stochastic resonance." In Communications (NCC), 2012 National Conference on, pp. 1-5. IEEE, 2012.

[8] Jadhav, B. D., and P. M. Patil. "Satellite Image Resolution Enhancement Using Dyadic-integer Coefficients Based Bi-orthogonal Wavelet Filters." Procedia Computer Science 49 (2015): 17-23.

[9] Xu, Hongteng, Guangtao Zhai, and Xiaokang Yang. "No reference measurement of contrast distortion and optimal contrast enhancement." InPattern Recognition (ICPR), 2012 21st International Conference on, pp. 1981-1984. IEEE, 2012.

[10] Verbesselt, Jan, Achim Zeileis, and Martin Herold. "Near real-time disturbance detection using satellite image time
International Journal of Computer Applications (0975 - 8887)

Volume 125 - No.13, September 2015

series." Remote Sensing of Environment123 (2012): 98108.

[11] Chouhan, Rajlaxmi, Rajib Kumar Jha, and Prabir Kumar Biswas. "Enhancement of dark and low-contrast images using dynamic stochastic resonance." IET Image Processing 7, no. 2 (2013): 174-184.

[12] Petitjean, François, Jordi Inglada, and Pierre Gançarski. "Satellite image time series analysis under time warping." Geoscience and Remote Sensing, IEEE Transactions on 50, no. 8 (2012): 3081-3095.

[13] Reshmalakshmi, C., and M. Sasikumar. "Image contrast enhancement using fuzzy technique." In Circuits, Power and Computing Technologies (ICCPCT), 2013 International Conference on, pp. 861-865. IEEE, 2013.

[14] Song, Weiwei, Dunwei Wen, Ke Wang, Tong Liu, and Mujun Zang. "Satellite image scene classification using spatial information." In Sixth International Conference on Graphic and Image Processing (ICGIP 2014), pp. 94431K-94431K. International Society for Optics and Photonics, 2015

[15] Maragatham, G., and S. Md Mansoor Roomi. "An automatic contrast enhancement method based on stochastic resonance." In 2013 Fourth International Conference on Computing, Communications and Networking Technologies (ICCCNT), pp. 1-7. IEEE, 2013.

[16] McRoberts, Ronald E. "Satellite image-based maps: Scientific inference or pretty pictures?." Remote Sensing of Environment 115, no. 2 (2011): 715-724.

[17] Cao, Gang, Yao Zhao, Rongrong Ni, and Xuelong Li. "Contrast enhancement-based forensics in digital images." Information Forensics and Security, IEEE Transactions on 9, no. 3 (2014): 515-525.

[18] Iqbal, Muhammad Zafar, Abdul Ghafoor, and Adil Masood Siddiqui. "Satellite image resolution enhancement using dual-tree complex wavelet transform and nonlocal means." Geoscience and Remote Sensing Letters, IEEE 10, no. 3 (2013): 451-455. 